\section{Time for a U-Turn on Understanding the Major Cause of Universal Human Hypovitaminosis D}

\section{Abstract}

Vitamin D deficiency has turned into a rather concerning health issue around the globe, touching at least $40-50 \%$ of general population. The old and stereotyped view with regard to the problem is that, the current hypovitaminosis $D$ results from inadequate vitamin $D$ in human diet and lack of enough sunlight exposure. And even more amusing is that the vast majority of recent epidemic human health problems are being somehow regarded as the direct outcome of this particular micronutrient deficiency. Our major aim in this brief overview, which will be followed by an opinion, is to cast a faint beam of light onto a narrow road towards a more sensible understanding of this utterly bizarre and poorly explained human health dilemma. We would like to raise the impression that the current vitamin $D$ deficiency state is not merely a simple dietary inadequacy, but an immediate outcome of metabolic syndrome and its closely related medical entity, the nonalcoholic fatty liver disease (NAFLD). We would like to put forward the idea of vitamin $D$ malabsorption and impaired 25 -hydroxylation of vitamin D precursors in subjects affected by NAFLD, and to open a wide field of research in front of dedicated and loyal scientists.

Keywords: Vitamin D deficiency; NAFLD; Vit D malabsorption; 25-hydroxylation defect

Received: May 28, 2018; Accepted: June 08, 2018; Published: June 13, 2018

\section{Abbas Tavakolian Arjmand ${ }^{1 *}$ and Jafarian $\mathrm{S}^{2}$}

1 Department of Internal Medicine, Diabetes \& Metabolic Disorders, Islamic Azad University (IAU), Shahrood branch, Shahrood, Iran

2 Department of pediatrics endocrinology and metabolism, Islamic Azad University (IAU), Shahrood branch, Shahrood, Iran

\section{*Corresponding author:}

Tavakolian Arjmand A

\section{ほdr.Tavakolian@gmail.com}

Department of Internal Medicine, Diabetes \& Metabolic Disorders, Islamic Azad University (IAU), Shahrood branch, Shahrood, Iran.

Tel: +982332392661

Fax: + 982332331876

Citation: Arjmand AT, Jafarian S (2018) Time for a U-Turn on Understanding the Major Cause of Universal Human Hypovitaminosis D. Ann Clin Lab Res. Vol.6 No.2: 238

\section{Introduction}

Human vitamin D requirement is supplied by two major sources: D2 (ergocalciferol) and D3 (cholecaciferol). Vitamin D3 is synthesized in skin via sun exposure and ultraviolet irradiation of the epidermis and vitamin D2 comes from food stuffs, especially beef liver, cheese, egg yolk and fatty fish. For longer half- life of 25- hydroxy vitamin $D$, compared to $1-25(\mathrm{OH})_{2}$, vitamin $D$, serum 25-OH-vitamin D has become the standard laboratory test to define vitamin D status. A serum $25(\mathrm{OH})$ vitamin D levels of $<20$ $\mathrm{ng} / \mathrm{ml}$ has been considered as vitamin $D$ deficiency, and values between $21-29 \mathrm{ng} / \mathrm{ml}$ as insufficiency. Vitamin $D$ is sufficient if it is $>30 \mathrm{ng} / \mathrm{ml}$ and in toxic levels if $>150 \mathrm{ng} / \mathrm{ml}$.

\section{Literature Review}

Vitamin D deficiency has evolved into a totally perplexing human health problem worldwide, affecting more than fifty percent of the general population. According to recent reports, the highest rate of $25(\mathrm{OH}) \mathrm{D}$ deficiency has been observed in blacks $(82 \%)$, followed by Hispanics and middle- east inhabitants (70\%). It would be quite a surprise to point out that, even the people living in countries with adequate sun exposure and high standards of living where most food stuffs are fortified with vitamin D, demonstrate incredibly high prevalence of $25(\mathrm{OH}) \mathrm{D}$ deficiency; an important subject which needs to be discussed comprehensively and questioned reasonably [1-4]. In a recently published study from Qatar, Al-Dabhani and his colleagues reported that $68 \%$ of men and $64 \%$ of women suffered from severe vitamin D deficiency. His work, as many other reports, revealed a strong association between metabolic syndrome and 25- hydroxy vitamin $D$ deficiency. The study also referred to another notable issue; the higher the waist circumference the lower the serum levels of $25(\mathrm{OH})$ vitamin D [5]. During the past three decades we have been continuously shuttling around a vicious cycle, considering the current hypovitaminosis $D$ as a nutritional shortage of vitamin $D$, or the lack of enough exposure to sun light. And farther than this, we have made an extensive 
effort to display a kind of cause and effect relationship between hypovitaminosis $D$ and a wide variety of disorders from up on the top of the head (baldness, headache, depression, benign intracranial hypertension) down to the tip of the toes (sensory neuropathy, toe nail dystrophy and...)! The shortest list of disorders suggested to be related to, or even presumed to be due to vitamin D deficiency would consist of: Metabolic Syndrome, obesity, high waist circumference, hypertriglyceridemia, low serum HDL-c, hypertension, atherosclerotic cardiovascular disease, PCOS, BPH, prostate cancer, breast cancer, T1DM, T2DM, multiple sclerosis, inflammatory bowel disease, SLE, RA, depression, Alzheimer's disease, hematologic disorders, migraine, tension type headache, benign intracranial hypertension, baldness, neuropathies, infertility, pregnancy loss and so on [6-16]. For instance, the cause and consequence relationship between hypovitaminosis $D$ and NAFLD has been taken so straightforward that in a recently published paper by Papapostoli and the co-workers, even fourweek vitamin D supplement resulted in significant NAFLD regression [17], or the report of rapid symptomatic relief of type1 diabetes neuropathy through correction of vitamin $D$ deficiency [18]. How to accept that almost all current human health- related issues directly stem from a simple micronutrient deficiency in the diet (vitamin D), and we, nevertheless, are standing stock-still doing nothing about it? The galloping incidence and the alarming prevalence of metabolic syndrome, obesity, NAFLD, T2DM and cardiovascular disease have put the human life on the edge of a certain catastrophe, and we simply address the whole problem to be merely on account of vitamin D shortage in human diet. The dramatic surge in infertility rate and pregnancy losses are turning into real threats towards the human generation, and we confidently blame low serum $25-\mathrm{OH}$ vitamin $\mathrm{D}$ levels as the main culprit and perhaps recommend beautifully packed vitamin $D$ containing pearls as the miraculous remedy! It looks as if, we have been facing with a big illusory bias in considering an innocent bystander (low serum $25 \mathrm{OH} \mathrm{D}$ ) as the putative cause for a host of human diseases. We might have failed to comprehend the depth of what has been happening to human over the past few decades. When two basically unrelated phenomena are highly prevalent in a defined cohort, it is not unusual to find an apparent association between them. Almost $50 \%$ of 35-year-old men are married in my society, and $50 \%$ of the same population is bald as well. Is it surprising to find a statistically significant association between marital status and male-pattern baldness in this particular cohort? Should this vivid association simply arouse the notion that marriage could be a powerful risk for male baldness? This delusive perception might have been happening in many studies trying to find a relationship between hypovitaminosis $D$ and the incredibly prevalent accompaniments of metabolic syndrome. What we are trying to reason out is that, the current low $25(\mathrm{OH})$ vitamin $\mathrm{D}$ epidemic might be considered as one of the countless disorders related to insulin resistance/ hyperinsulinemia syndrome. We believe that current vitamin $D$ deficiency actually emerged concurrent with a poorly explained insulin resistance flare- up in late 1980s, which then rapidly turned into a worldwide health catastrophe defined as metabolic syndrome. It is worth noting that, the prevalence of the three major human health problems today (metabolic syndrome, NAFLD and vitamin D deficiency) is exactly the same, roughly $50 \%$, and their pathophysiologic processes are so interlaced that determination of the cause and definition of the consequence has changed to a major dilemma, and a source of gross delusive biases in research in due course. To summarize the entire pathophysiology of metabolic syndrome in a few short sentences, one might say that, a poorly described, probably an environmental factor, brought about an overwhelming insulin resistance state in human being. The very first and foremost response of the body and perhaps the sole chance to cope with this tumultuous state was a correspondent increase in pancreatic B-cell insulin production; an unwanted and undesirable response which gave way to an immense concentrations of insulin in the portal vein and to lesser extent, in systemic circulation. At the top on the list of undesirable effects of portal vein insulin plethora, stand the superfluous synthesis of de novo fatty acid, Apo B100, and triglyceride-rich VLDL particles by the liver cells. In such a chaotic situation, Non-alcoholic fatty liver disease begins with gradual accumulation of VLDL-containing lipid droplets inside the liver cells, and progresses towards a deleterious cellular oxidative stress, accumulation of free radicals, inflammatory reactions, $\mathrm{NASH}$, fibrosis, occult cryptogenic cirrhosis and eventually overt impairment in almost all synthetic and secretory functions of the liver, not to mention the delicate process of bile synthesis, bile modification and secretion [19-33]. The first and foremost hepatic commitment towards thorough intestinal absorption of dietary fat and fat soluble constituents, especially fat soluble vitamins, including vitamin $D$, is to provide the duodenum with a generous amount of high quality bile. The organic and inorganic solutes of bile are of considerable complexity. Being deeply engaged in the fine process of fat emulsification, bile is considered as the sine qua non for proper fat digestion and absorption, without which a serious malabsorption of dietary fat will take place. Even minor failure in this vital task of bile will lead to surreptitious defect in absorption of fat and fat-soluble vitamin $D$ in due course $[34,35]$. Considering what was briefly described, it seems quite logical to suggest that, in NAFLD of metabolic syndrome, the secretion of poor quality bile could simply give rise to a surreptitious fat malabsorption and undercover disturbance in vitamin $D$ assimilation from the small intestine. That is why from all reports concerning the hypovitaminosis $D$ associations so far, the relationship between NAFLD and 25(OH) $D$ deficiency has received the highest strength, remaining significant enough even independent of metabolic syndrome, obesity, diabetes and insulin resistance profile. But, this is not the end of the story yet. In addition to mentioned possibility of vitamin D malabsorption, progressive liver cell dysfunction might negatively affect the hepatic 25 - hydroxylation capacity, causing a state of low serum $25(\mathrm{OH})$ D despite near-normal serum vitamin D2 and D3 concentrations. Thus, in NAFLD, the low serum levels of $25(\mathrm{OH}) \mathrm{D}$ might be the outcome of two NAFLD related phenomena; occult vitamin $\mathrm{D}$ malabsorption and defective 25 hydroxylation of vitamin D precursors (Figure 1).

Stringent and inflexible low fat diet prescribed by health providers, 


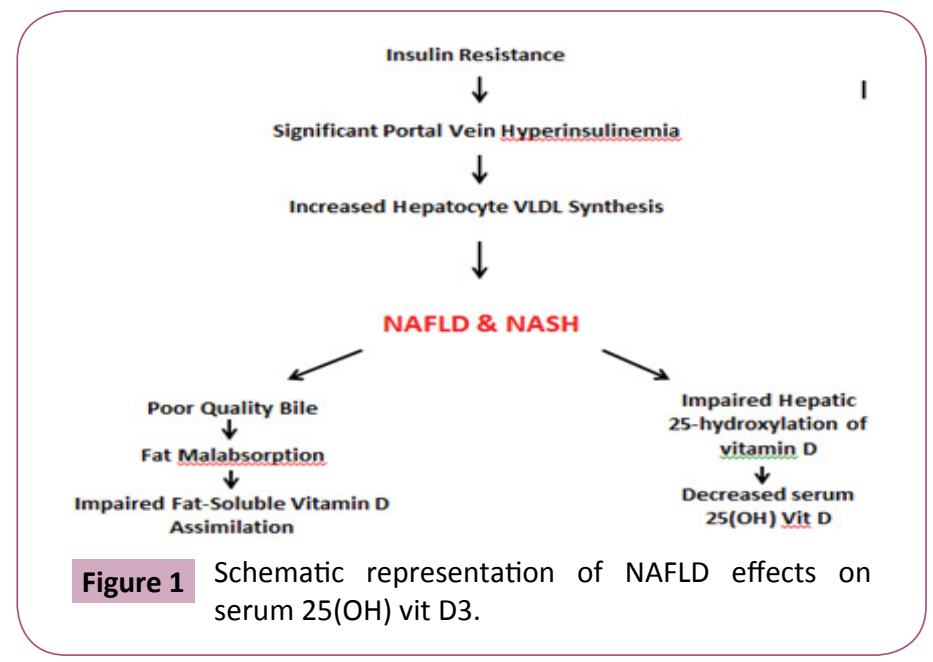

being stressed with great enthusiasm by the media as well, can also contribute as an auxiliary risk for vitamin $D$ malabsorption. It was noted that vitamin D is a strict fat- soluble substance. Hence, enough fat in ingested meal is, indeed, a prerequisite for effective assimilation of vitamin $\mathrm{D}$.

The issue of vitamin D storage in adipose tissue is also worth noting. Although negligible in thin healthy subjects, but the abnormally expanded adipose tissue of metabolic syndrome, could soak up a notable portion of serum vitamin D2, D3, and their 25-hydroxylated form, accounting for another presumptive factor for current low serum $25(\mathrm{OH}) \mathrm{D}$. Now one might gather the core concept behind the negative correlation between high waist circumference and low serum $25(\mathrm{OH}) \mathrm{D}$. On a setting of insufficient vitamin $\mathrm{D}$ absorption and defective 25-hydroxylation, the increased storage capacity of expanded visceral adipose tissue might receive further importance.

\section{Discussion and Conclusion}

Contrary to current belief, we would like to raise the impression that vitamin $D$ deficiency state is not merely a simple dietary

\section{References}

1 Holick MF, Chen TC (2008) Vitamin D deficiency: A worldwide problem with health consequences. Am J Clin Nutr 87(4): 1080-1086.

2 Mithal A, Wahl DA, Bonjour JP, Burkhardit P, Dawson-Hughes B, et al. (2009) IOF Committee of Scientific Advisors (CSA) nutrition working group, global vitamin $D$ status and determinants of hypovitaminosis D. Osteoporosis Int 20(11): 1807-1820.

3 Van Schoor NM, lips P (2011) Worldwide vitamin D status. Best Pract Res Clin Endocrinol Metab 25(4): 671-680.

4 Forrest KY, Stuhdreher WL (2001) Prevalence and correlates of vitamin D deficiency in US adults. Nutr Res 31(1): 48-54.

5 Al-Dabhani K, Tsilidis KK, Morphy N, Elliot HA (2017) Prevalence of vitamin $\mathrm{D}$ deficiency and association with metabolic syndrome in a Qatari population. Nutrition \& Diabetes 7: e263.

6 Houriqan SK, Abrams S Yatesk, Peifer K (2015) Relation between inadequacy, but an immediate outcome of metabolic syndrome and its closely related attendant, the non-alcoholic fatty liver disease (NAFLD). We would like to put forward the idea of vitamin $D$ malabsorption and impaired 25 hydroxylation of vitamin $D$ as the two major operating factors with regard to the currently observed low serum 25- hydroxy vitamin D levels in human.

\section{Future Directions and Recommendations}

Taking into consideration the above mentioned sensible opinion on pathogenesis of hypovitaminosis $D$, we should set out to investigate the quality of bile and the possibility of its undercover role on fat and fat-soluble vitamin D malabsorption in subjects suffering from NAFLD, and probe the likelihood of hepatic failure in 25 hydroxylation of absorbed vitamin $D$. If our suggestions prove useful, the treatment of low serum 25-hydroxy vitamin $D$ should be based upon proper management of metabolic syndrome and its closely related disorder that is NAFLD, instead of being totally focused on dietary deficiency or lack of enough sun light as the sole putative cause of serum vitamin D shortage.

\section{Conflict of Interest}

No conflicts of interest to be declared.

\section{Authors' contribution profile}

Tavakolian Arjmand A wrote the paper and is responsible for final content. Jafarian $S$ carried out an extensive literature review especially in the field of childhood and adolescent vitamin D deficiency.

\section{Acknowledgements}

Our sincere appreciations go to thousands of devoted senior researchers whose valuable works made a concrete platform for this paper to be launched so smooth. We would like to thank the Azad University of Shahrood authorities for their administrative and managerial services as well.

vitamin D status and nonalcoholic fatty liver disease in children. $J$ Pediat Gastroentrol Nutr 60(3): 396-404.

7 Barchetta I, Angolico F, Delben M, Baroni MG, Pozzilli P, et al. (2011) Strong association between nonalcoholic fatty liver disease (NAFLD) and low $25(\mathrm{OH})$ vit $D$ levels in an adult population with normal serum enzymes. BMC Med 9: 1741-7045.

8 Espinosa G, Esposito R, Kazzazi A, Djavan B (2013) Vitamin D and prostatic hyperplasia-areview. Can J Urol 20(4): 6820-6825.

9 Zittermann A, Gummert JF, Borgermann J (2011) The role of vit D in dyslipidemia and cardiovascular disease. Curr Pharm Des 17(9): 933-942.

10 Suzanne E, Judd, Vin Tangpricha (2009) Vitamin D deficiency and risk of cardiovascular disease. Am J Med Sci 338(1): 40-44.

11 Mehta V, Agarwal S (2017) Does vitamin D deficiency lead to htpertension? Cureus 9(2): e1036.

12 Witham MD, Nadir MA, Struthers AD (2013) Effect of vitamin D 
on blood pressure: A systematic review and meta-analysis. Eur J Epidemiol 28: 205-221.

13 Lin MW, Wu MH (2015) The role of vitamin D in polycystic ovary syndrome. Indian J Med Res 142(3): 238-240.

14 Irani M, Merhi Z (2014) Role of vitamin D in ovarian physiology and its implication in reproduction: A systemic review. Fertil Steril 102 460- 468.

15 Wakayo T, Whitting SJ, Belachew T (2016) Vitamin D deficiency is associated with overweight and/or central Ethiopia: A cross-sectional study. Nutrients 8(4): 190.

16 Kavaric S, Vuksanovic M, Bozovic D, Jovanovic $M$, Jeremic V, et al. (2013) Body weight and waist circumference as predictors of vitamin D deficiencyin patients with type 2 diabetes and cardiovascular disease. Vajnosanit Pregl 70(2): 163-169.

17 Papapostoli L, Lammert F, Stokes CS (2016) Effect of short-term vitamin $\mathrm{D}$ correction on hepatic steatosis as quantified by controlled attenuation parameter(CAP). J Gastrointestin Liver Dis 25(2): 175-181.

18 David SH, Bell (2012) Reversal of the symptoms of diabetic neuropathy through correction of vit $D$ deficiency in type 1 diabetes patients. Case Rep Endocrinol p: 165056.

19 Tavakolian Arjmand A (2016) A novel look into pathogenesis and a logical hint towards the etiology of metabolic syndrome: The jigglysignal and wiggly-receptor hypothesis. Int J Diabetes Metab Disord 1(2): 1-6.

20 Botella-Carretero JI, Alvarez-Blasso F, Villafruella JJ, Jose A Balsa (2007) Vitamin D deficiency is associated with the metabolic syndrome in morbid obesity. Clinical Nutrition 26(5): 573-580.

21 Strange RC, Shipman KE, Amachandran SR (2015) Metabolic syndrome: A review of the role of vitamin $D$ in mediating susceptibility and outcome. World J Diabetes 6(7): 896-911.

22 Awad AB, Alappat L, Valerio M (2012) Vitamin D and metabolic syndrome risk factor: Evidence and mechanism. Crit Rev Food Sci Nutr 52(2): 103-112.

23 Tavakolian Arjmand A, Nouri M, Tavakolian Arjmand S (2015) Surprisingly low infertility rate in married type 2 diabetic women: A rather curious paradox to the current opinion of insulin resistance as the joint pathogenesis of polycystic ovary syndrome and type 2 diabetes. Diab \& Metab syndr: Cli Res \& Rev 9(4): 201-205.

24 Choi SH, Ginsberg HN (2011) Increased very low-density
lipoprotein(VLDL) secretion in hepatic steatosis and insulin resistance. Trends Endocrinol Metab 22(9): 353-363.

25 Fabrini E, Mohammed BS, Maykos F, Korenblat KM (2008) Alterations in adipose tissue and hepatic lipid kinetics in obese men and women with nonalcoholic fatty liver disease. Gastroentrology 134: 428-431.

26 Lewis GF, Uffelman KD, Szeto Weller B, Steiner G (1995) Interaction between free fatty acids and insulin in the acute control of very lowdensity lipoprotein in human. J Clin Invest 95: 158-166.

27 Castro Cabezas M, De Bruin TW, De Valk HW, Shoulders CC, James $\mathrm{H}$, et al. (1993) Impaired fatty acid metabolism in familial combined hyperlipidemia: A mechanism associating hepatic apolipoprotein B overproduction and insulin resistance. J Cli Invest 92: 160-168.

28 Younosi ZM, Koenig AB, Abdelatif D, Fazel Y, Henry L (2016) Global epidemiology of nonalcoholic fatty liver disease, meta-analytic assessment of prevalence, incidence, and outcomes. Hepetology 64(1): 73-84.

29 Miquilena-Colina ME, Lima-Cabello E, Sanchez-Cops S, GarciaMediavilla MN (2011) Hepatic fatty acid translocase CD36 upregulation is associated with insulin resistance, hyperinsulinemia and steatohepatitis and increased steatosis in nonalcoholic steatohepatitis and chronic hepatitis C Gut p: 26.

30 Postic C, Girard J (2018) Contribution of de novo fatty acid synthesis to hepatic steatosis and insulin resistance: Lessons from genetically engineered mice. J Cli Invest 118: 829-838.

31 Taghibiglou C, Carpentier A, C Van Iderstein S, Aiton A, Gary F, et al. (2000) Mechanisms of hepatic very low-density lipoprotein overproduction in insulin resistance. The Journal of Biological Chemistry 275: 8416-8425.

32 Tavakolian Arjmand A, Razavianzadeh N (2016) The cause and effect relationship between type 2 diabetes and clinically overt cryptogenic cirrhosis: A matter that must be seriously revised. Hept Mon 16(9): e36485.

33 Black LJ, Jacoby $P$, She Ping-Delfo WC, Mori TA, Beilin LJ, et al. (2014) Low serum25-hydroxy vitamin D concentrations associate with nonalcoholic fatty liver disease in adolescents independent of adiposity. J Gastroentrol 29(6): 1215-1222.

34 Boyer JL (2013) Bile formation and secretion. Compr Physiol 65(1): 350-362.

35 Arab JP, Karpen SJ, Dawson P, Arrese M (2017) Bile acid and nonalcoholic fatty liver disease: Molecular insights and therapeutic perspectives. Hepatology 65(1): 350-362. 